\title{
Correction to: Management of Patient with Brachial Plexus Injury
}

Hossam El Beheiry

\section{Correction to: H. Prabhakar et al. (eds.), Problem Based Learning Discussions in Neuroanesthesia and Neurocritical Care, https://doi.org/10.1007/978-981-15-0458-7}

In chapter 2, the abstract content was inadvertently duplicated as first paragraph of the chapter in the original version of this book. It has been corrected now and duplication of abstract content was removed and updated with correct stem cells content. 